\title{
A SPACE-TIME DISCONTINUOUS GALERKIN METHOD FOR FIRST ORDER HYPERBOLIC SYSTEMS
}

\author{
Tie Zhang AND Jingna LiU
}

\begin{abstract}
We present a new space-time discontinuous Galerkin (DG) method for solving the time dependent, positive symmetric hyperbolic systems. The main feature of this DG method is that the discrete equations can be solved semi-explicitly, layer by layer, in time direction. For the partition made of triangle or rectangular meshes, we give the stability analysis of this DG method and derive the optimal error estimates in the DG-norm which is stronger than the $L_{2}$-norm. As application, the wave equation is considered and some numerical experiments are provided to illustrate the validity of this DG method.
\end{abstract}

\section{Introduction}

During the last decades, the discontinuous Galerkin (DG) finite element methods have attracted more and more attention in the field of numerical partial differential equations, see $[1,3,5,6,14]$ and the references therein. In this paper, we will consider the space-time DG method for solving the timedependent, positive symmetric hyperbolic systems. Traditionally, for timedependent problems, the fully discrete finite element methods are constructed by using finite elements to discretize in space, but using finite difference or other methods to discretize in time. The disadvantage of this kind of discrete methods is that it is difficult to enhance the approximation accuracy in time direction. However, this shortcoming can be overcome by adopting the space-time finite element methods. Since 1980s, some explicit and semi-explicit (in time) spacetime finite element methods have been presented for first order hyperbolic systems. Winther in [13] first gave an explicit scheme, but it is restricted to one space dimension. Later, Johnson, et al. in [9] proposed the semi-explicit method by using continuous finite elements in space and discontinuous trial functions in time. Recently, Falk and Richter [7] construct a space-time DG

Received May 17, 2013; Revised February 24, 2014.

2010 Mathematics Subject Classification. Primary 65N12, 65N30, 65M60.

Key words and phrases. discontinuous Galerkin method, first-order hyperbolic system, semi-explicit scheme, stability and error estimate.

The work is supported by the National Natural Science Funds of China (11371081) and the SAPI Fundamental Research Funds (2013ZCX02). 
method for hyperbolic systems, and this method is further developed by Monk and Richter in [10]. The main feature of this two space-time DG methods is that they are explicit or semi-explicit in time-direction. But, this two methods are only available to the structured triangle meshes. More specifically, let $n_{t}$ and $n_{x}=\left(n_{1}, \ldots, n_{d}\right)^{T}$ be the time and spatial components of the unit outward normal vector $\mathbf{n}=\left(n_{t}, n_{x}\right)^{T}$ in the space-time domain $\Omega_{T}$, respectively, and $\mathcal{T}_{h}$ be the regular triangulation of domain $\Omega_{T}$. The structured triangle meshes used in $[7,10]$ requires the key mesh condition, for some $\varepsilon_{0}$ properly small,

$$
\frac{\left|n_{x}(t, x)\right|}{\left|n_{t}(t, x)\right|} \leq \varepsilon_{0}, \forall(t, x) \in \partial K \backslash \partial \Omega_{T} \text { or } \partial K^{*} \backslash \partial \Omega_{T},
$$

where $K \in \mathcal{T}_{h}$ is the space-time element (for the explicit method) and $K^{*}$ is the micro element in $\mathcal{T}_{h}$ (for the semi-explicit method). It is easy to see that condition (1.1) implies a time-step (CFL) constraint on the ratio $\triangle t / \triangle x$, where $\triangle t$ and $\triangle x$ are the mesh steps in the time-direction and space-direction, respectively. Moreover, condition (1.1) can not be satisfied by the rectangular meshes, in this case, for each $K$ or $K^{*}$, there exists always a face $\mathcal{F}_{K} \in \partial K$ (or $\left.\partial K^{*}\right)$ so that $\left.n_{t}\right|_{\mathcal{F}_{K}}=0$.

The goal of this paper is to present a new space-time DG method for solving the time-dependent, positive symmetric hyperbolic systems. For appropriate shape-regular triangulations (including rectangular meshes) without restriction condition (1.1), we construct a semi-explicit DG scheme by elaborately designing the numerical traces on the element interfaces. The main feature of this DG method is that the discrete equations can be solved semi-explicitly, layer by layer, in time direction. We give the stability and error analysis for the DG solution, and derive the optimal error estimates of order $k+1 / 2$ in the DG-norm if piecewise polynomials of degree $k$ are used.

Throughout this paper, let $\Omega$ be a bounded open polyhedral domain in $\mathbb{R}^{d}$, $d \geq 1$, the space-time domain $\Omega_{T}=(0, T] \times \Omega$. For any open subset $\mathcal{D} \subset \Omega_{T}$ and integers $m \geq 0$, we denote by $H^{m}(\mathcal{D})$ the usual Sobolev spaces equipped with norm $\|\cdot\|_{m, \mathcal{D}}$ and semi-norm $|\cdot|_{m, \mathcal{D}}$, and denote by $(\cdot, \cdot)_{\mathcal{D}}$ and $\|\cdot\|_{\mathcal{D}}$ the standard inner product and norm in the space $H^{0}(\mathcal{D})=L_{2}(\mathcal{D})$. When $\mathcal{D}=\Omega_{T}$, we omit the index $\mathcal{D}$. We will also use letter $C$ to represent a generic positive constant, independent of the mesh size $h$.

The plan of this paper is as follows. In Section 2, the DG method is constructed. In Section 3, the stability and semi-explicit structure of this DG method are analyzed. Section 4 is devoted to the optimal error estimates in the DG-norm. Finally, in Section 5, we provide some numerical experiments applied to the wave equation to illustrate the validity of our method.

\section{Problem and its DG approximation}

Let $\Omega$ be a bounded polyhedral domain in $\mathbb{R}^{d}, d \geq 1$, and $\mathbf{u}=\left(u_{1}, \ldots, u_{m}\right)^{T}$ denote the $m$-vector function on the space-time domain $\Omega_{T}=(0, T] \times \Omega$. Consider the following time-dependent first-order hyperbolic system: 


$$
\begin{aligned}
A_{0} \partial_{t} \mathbf{u}+\sum_{k=1}^{d} A_{k} \partial_{k} \mathbf{u}+B \mathbf{u} & =\mathbf{f}, \quad(t, x) \in(0, T] \times \Omega, \\
(M-D) \mathbf{u} & =\mathbf{0},(t, x) \in(0, T] \times \partial \Omega, \\
\mathbf{u}(0) & =\mathbf{u}_{0}, x \in \Omega .
\end{aligned}
$$

Here, $A_{0}$ is a positive definite and symmetric matrix, independent of time $t$ (typically $\left.A_{0}=I\right), A_{k}=\left(a_{i j}^{(k)}(x)\right), B=\left(b_{i j}(x)\right)$ and $M=\left(m_{i j}(x)\right)$ are some given $m \times m$ matrices, $D=\sum_{k=1}^{d} A_{k} n_{k}$, and $n_{x}=\left(n_{1}, \ldots, n_{d}\right)^{T}$ is the spatial component of the outward unit normal vector $\mathbf{n}=\left(n_{t}, n_{x}\right)^{T}$ on $\partial \Omega_{T}$. We assume that problem (2.1)-(2.3) is a positive and symmetric hyperbolic system [8], namely,

$$
\begin{aligned}
& A_{k}=A_{k}^{T}, k=1, \ldots, d, x \in \Omega, \\
& B+B^{T}-\sum_{k=1}^{d} \partial_{k} A_{k} \geq 2 \sigma_{0} I, x \in \Omega, \\
& M+M^{T} \geq 0, x \in \partial \Omega, \\
& \operatorname{Ker}(M-D)+\operatorname{Ker}(M+D)=R^{m}, x \in \partial \Omega,
\end{aligned}
$$

where constant $\sigma_{0}>0$, and by using the expression $A \geq 0(A>0)$ we imply that matrix $A$ is positive semi-definite (positive definite). In what follows, we assume that the matrix elements $a_{i j}^{(k)}(x), b_{i j}(x)$ and $m_{i j}(x)$ are sufficiently smooth and bounded.

Remark 2.1. For the linear time-dependent problem (2.1), condition (2.5) is not essential. In fact, we always can use transformation $\mathbf{u}=e^{\sigma t} \mathbf{u}$ in equation (2.1) with $\sigma>0$ properly large such that condition (2.5) holds naturally.

Problem (2.1)-(2.3) can describe many important physics processes. An example of such positive and symmetric hyperbolic system is as follows.

Wave equation. Consider the wave equation in $R^{2}$

$$
\begin{aligned}
u_{t t}-\triangle u & =f, \quad(t, x) \in(0, T] \times \Omega, \\
u & =0,(t, x) \in(0, T] \times \partial \Omega, \\
u(0, x) & =\varphi, u_{t}(0, x)=\phi, \quad x \in \Omega,
\end{aligned}
$$

where $\Omega \subset R^{2}$ is a bounded domain. Introduce the transformation: $u_{0}=$ $\partial_{t} u, u_{1}=\partial_{1} u, u_{2}=\partial_{2} u$, then we have the equivalent equations

$$
\frac{\partial u_{0}}{\partial t}-\frac{\partial u_{1}}{\partial x_{1}}-\frac{\partial u_{2}}{\partial x_{2}}=f, \quad \frac{\partial u_{1}}{\partial t}-\frac{\partial u_{0}}{\partial x_{1}}=0, \frac{\partial u_{2}}{\partial t}-\frac{\partial u_{0}}{\partial x_{2}}=0 .
$$

Now, the wave problem (2.8) can be written as the following positive and symmetric hyperbolic system (setting $\mathbf{u}=\left(u_{0}, u_{1}, u_{2}\right)^{T}$ ),

$$
\begin{aligned}
\partial_{t} \mathbf{u}+A_{1} \partial_{1} \mathbf{u}+A_{2} \partial_{2} \mathbf{u} & =\mathbf{f}, \quad(t, x) \in(0, T] \times \Omega, \\
(M-D) \mathbf{u} & =\mathbf{0},(t, x) \in(0, T] \times \partial \Omega
\end{aligned}
$$


with the initial value $\mathbf{u}_{0}=\left(\phi, \partial_{1} \varphi, \partial_{2} \varphi\right)^{T}$, vector function $\mathbf{f}=(f, 0,0)^{T}$, and matrices

$A_{0}=\left(\begin{array}{lll}1 & 0 & 0 \\ 0 & 1 & 0 \\ 0 & 0 & 1\end{array}\right), A_{1}=\left(\begin{array}{ccc}0 & -1 & 0 \\ -1 & 0 & 0 \\ 0 & 0 & 0\end{array}\right), A_{2}=\left(\begin{array}{ccc}0 & 0 & -1 \\ 0 & 0 & 0 \\ -1 & 0 & 0\end{array}\right), B=0$,

and choosing the boundary matrix

$$
M=\left(\begin{array}{ccc}
1 & -n_{1} & -n_{2} \\
n_{1} & 0 & 0 \\
n_{2} & 0 & 0
\end{array}\right), \quad M-D=\left(\begin{array}{ccc}
1 & 0 & 0 \\
2 n_{1} & 0 & 0 \\
2 n_{2} & 0 & 0
\end{array}\right) .
$$

The conditions (2.4)-(2.7) can be verified directly.

In the above example, although the boundary matrix $M$ should be determined by the boundary value condition of the problem, it is not unique. In this example, we have chosen the boundary matrix $M$ properly such that it also satisfies our requirement for the error analysis, see (4.16).

Now let us introduce the space-time DG method for solving the problem (2.1)-(2.3). Let $\mathcal{T}_{h}=\bigcup\{K\}$ be a shape regular partition of the space-time domain $\Omega_{T}$ parameterized by mesh size $h=\max h_{K}$ so that $\bar{\Omega}_{T}=\bigcup_{K \in \mathcal{T}_{h}}\{\bar{K}\}$, where $h_{K}$ is the diameter of element $K$. We say that the partition $\mathcal{T}_{h}$ is shape reqular, if the elements of $\mathcal{T}_{h}$ are affine and there exists a positive constant $\gamma$ independent of $K \in \mathcal{T}_{h}$ such that

$$
h_{K} / \rho_{K} \leq \gamma, \forall K \in \mathcal{T}_{h},
$$

where $\rho_{K}$ denotes the diameter of the biggest ball included in $K$. To partition $\mathcal{T}_{h}$, we associate the finite-dimensional space $V_{h}$,

$$
V_{h}=\left[S_{h}\right]^{m}, S_{h}=\left\{v \in L_{2}\left(\Omega_{T}\right):\left.v\right|_{K} \in S_{k}(K), \forall K \in \mathcal{T}_{h}\right\},
$$

where $S_{k}(K)$ is the local finite element space composed of polynomials which at least includes $P_{k}(K)$. Typically, $S_{k}(K)$ is the space $P_{k}(K)$ of polynomials of degree at most $k$ on $K$ for triangle meshes, or the space $Q_{k}(K)$ of polynomials of degree at most $k$ in each variable on $K$ for rectangular meshes. We denote by $\mathcal{E}_{h}=\bigcup\left\{\partial K: K \in \mathcal{T}_{h}\right\}$ the union of all boundaries of elements.

Denote the piecewise smooth function space on $\mathcal{T}_{h}$ by

$$
H^{s}\left(\mathcal{T}_{h}\right)=\left\{v \in L_{2}\left(\Omega_{T}\right):\left.v\right|_{K} \in H^{s}(K), \forall K \in \mathcal{T}_{h}\right\}, s \geq 1 .
$$

In order to cope with the discontinuity of function across the interfaces of elements, we introduce the jump of function $\phi \in H^{1}\left(\mathcal{T}_{h}\right)$ on $\partial K$ by

$$
[\phi]=\phi^{+}-\phi^{-},\left.\phi^{-}\right|_{\partial \Omega_{T}}=0,
$$

where $\phi^{+}$and $\phi^{-}$are the traces of $\phi$ on $\partial K$ from the interior and exterior of $K$, respectively. Sometimes, for convenience, we will denote $\phi^{+}$by $\phi$ on $\partial K$. We will also use the discrete inner product notations

$$
(u, v)_{\Omega_{T}^{\Delta}}=\sum_{K \in \Omega_{T}^{\Delta}}(u, v)_{K}=\sum_{K \in \Omega_{T}^{\Delta}} \int_{K} u v d K,\langle u, v\rangle_{\mathcal{S}}=\sum_{\partial K \in \mathcal{S}} \int_{\partial K} u v d s,
$$


where $\Omega_{T}^{\Delta}$ is a subset of $\mathcal{T}_{h}$ and $\mathcal{S}$ is a subset of $\mathcal{E}_{h}$.

In order to define the semi-explicit space-time DG scheme, we need to introduce some notations. First, we divide the boundary $\partial \Omega_{T}$ into three parts:

$$
\Gamma_{0}=\left\{(t, x) \in \partial \Omega_{T}: n_{t}=0\right\} ; \Gamma_{ \pm}=\left\{(t, x) \in \partial \Omega_{T}: n_{t}= \pm 1\right\} .
$$

Obviously, $\Gamma_{0}=[0, T] \times \partial \Omega, \Gamma_{+}=\{t=T\} \times \Omega, \Gamma_{-}=\{t=0\} \times \Omega$. Introduce the partial differential operator and its adjoint form (noting that $\partial_{t} A_{0}=0$ )

$$
\mathcal{L}_{t}=A_{0} \partial_{t}+\sum_{k=1}^{d} A_{k} \partial_{k}+B, \quad \mathcal{L}_{t}^{*}=-A_{0} \partial_{t}-\sum_{k=1}^{d} A_{k} \partial_{k}+B^{T}-\sum_{k=1}^{d} \partial_{k} A_{k} .
$$

By using integration by parts, we have

$$
\int_{K} \mathcal{L}_{t} \mathbf{u} \cdot \mathbf{v}=\int_{K} \mathbf{u} \cdot \mathcal{L}_{t}^{*} \mathbf{v}+\int_{\partial K} \mathcal{N}_{\mathbf{n}} \mathbf{u} \cdot \mathbf{v}, \forall K \in \mathcal{T}_{h},
$$

where the boundary matrix $\mathcal{N}_{\mathbf{n}}=A_{0} n_{t}+D, D=\sum_{k=1}^{d} A_{k} n_{k}$. It easy to see that

$$
\left.\mathcal{N}_{\mathbf{n}}\right|_{\Gamma_{0}}=D,\left.\mathcal{N}_{\mathbf{n}}\right|_{\Gamma_{ \pm}}= \pm A_{0},\left.D\right|_{\Gamma_{ \pm}}=0 .
$$

Introduce the bilinear form

$$
\begin{aligned}
a_{K}(\mathbf{u}, \mathbf{v})= & \left(\mathcal{L}_{t} \mathbf{u}, \mathbf{v}\right)_{K}+\frac{1}{2}\left\langle\left(A_{0}-\mathcal{N}_{\mathbf{n}}\right)[\mathbf{u}], \mathbf{v}\right\rangle_{\partial K \backslash \Gamma_{0}} \\
& +\frac{1}{2}\langle(M-D) \mathbf{u}, \mathbf{v}\rangle_{\partial K} \cap \Gamma_{0}, \quad K \in \mathcal{T}_{h} .
\end{aligned}
$$

Let $\mathbf{u} \in\left[H^{1}(\Omega)\right]^{m}$ be the solution of problem (2.1)-(2.3), from (2.14) we see that $\mathbf{u}$ satisfies the following weak form

$$
a_{K}(\mathbf{u}, \mathbf{v})=(\mathbf{f}, \mathbf{v})_{K}+\left\langle A_{0} \mathbf{u}_{0}, \mathbf{v}\right\rangle_{\partial K} \cap \Gamma_{-}, \forall K \in \mathcal{T}_{h}, \mathbf{v} \in\left[H^{1}\left(\mathcal{T}_{h}\right)\right]^{m}
$$

Motivated by this weak formula, we define the DG approximation of problem (2.1)-(2.3) by finding $\mathbf{u}_{h} \in V_{h}$, restricted to $K \in \mathcal{T}_{h}$, such that

$$
a_{K}\left(\mathbf{u}_{h}, \mathbf{v}_{h}\right)=\left(\mathbf{f}, \mathbf{v}_{h}\right)_{K}+\left\langle A_{0} \mathbf{u}_{0}, \mathbf{v}\right\rangle_{\partial K} \cap \Gamma_{-}, \forall K \in \mathcal{T}_{h}, \mathbf{v}_{h} \in V_{h} .
$$

\section{Stability analysis of the DG method}

In this section, we will discuss the semi-explicit structure of discrete problem (2.17) under appropriate partition condition and give the stability analysis.

For $0 \leq t_{-}<t_{+} \leq T$, let $\Omega_{t}^{\Delta}=\left(t_{-}, t_{+}\right] \times \Omega \subset \Omega_{T}$ be a subdividing domain composed of some elements of $\mathcal{T}_{h}$ and its boundary $\partial \Omega_{t}^{\Delta}=\Gamma_{0}\left(\Omega_{t}^{\Delta}\right) \bigcup \Gamma_{ \pm}\left(\Omega_{t}^{\Delta}\right)$, where $\Gamma_{0}\left(\Omega_{t}^{\Delta}\right)=\bigcup\left\{\partial K \bigcap \Gamma_{0}: K \in \Omega_{t}^{\Delta}\right\}$ and $\Gamma_{ \pm}\left(\Omega_{t}^{\Delta}\right)=\left\{(t, x) \in \partial \Omega_{t}^{\Delta}: n_{t}=\right.$ $\pm 1\}$. Now, summing (2.17) for $K \in \Omega_{t}^{\Delta}$, we obtain the expression of discrete problem (2.17) on $\Omega_{t}^{\Delta}$ : Find $\mathbf{u}_{h} \in V_{h}$ such that

$$
a_{\Omega_{t}^{\Delta}}\left(\mathbf{u}_{h}, \mathbf{v}_{h}\right)=\left(\mathbf{f}, \mathbf{v}_{h}\right)_{\Omega_{t}^{\Delta}}+\left\langle A_{0} \mathbf{u}_{0}, \mathbf{v}_{h}\right\rangle_{\Gamma_{-}\left(\Omega_{t}^{\Delta}\right) \cap \Gamma_{-}}, \forall \mathbf{v}_{h} \in V_{h},
$$

where

$$
a_{\Omega_{t}^{\Delta}}(\mathbf{u}, \mathbf{v})=\sum_{K \in \Omega_{t}^{\Delta}} a_{K}(\mathbf{u}, \mathbf{v})
$$




$$
\begin{aligned}
= & \left(\mathcal{L}_{t} \mathbf{u}, \mathbf{v}\right)_{\Omega_{t}^{\Delta}}+\frac{1}{2} \sum_{K \in \Omega_{t}^{\Delta}}\left\langle\left(A_{0}-\mathcal{N}_{\mathbf{n}}\right)[\mathbf{u}], \mathbf{v}\right\rangle_{\partial K \backslash \Gamma_{0}} \\
& +\frac{1}{2} \sum_{K \in \Omega_{t}^{\Delta}}\langle(M-D) \mathbf{u}, \mathbf{v}\rangle_{\partial K} \cap \Gamma_{0} .
\end{aligned}
$$

We first give a useful lemma.

Lemma 3.1. Bilinear form $a_{\Omega_{t}^{\Delta}}(\mathbf{u}, \mathbf{v})$ satisfies the following identity.

$$
\begin{aligned}
a_{\Omega_{t}^{\Delta}}(\mathbf{w}, \mathbf{w})= & \frac{1}{2}(Q \mathbf{w}, \mathbf{w})_{\Omega_{t}^{\Delta}}+\frac{1}{2}\langle M \mathbf{w}, \mathbf{w}\rangle_{\Gamma_{0}\left(\Omega_{t}^{\Delta}\right)} \\
& +\frac{1}{4} \sum_{K \in \Omega_{t}^{\Delta}}\left\langle A_{0}[\mathbf{w}],[\mathbf{w}]\right\rangle_{\partial K \backslash \partial \Omega_{t}^{\Delta}}+\frac{1}{2}\left\langle A_{0} \mathbf{w}, \mathbf{w}\right\rangle_{\Gamma_{+}\left(\Omega_{t}^{\Delta}\right)} \\
& +\frac{1}{2}\left\langle A_{0} \mathbf{w}, \mathbf{w}\right\rangle_{\Gamma_{-}\left(\Omega_{t}^{\Delta}\right)}-\left\langle A_{0} \mathbf{w}^{-}, \mathbf{w}\right\rangle_{\Gamma_{-}\left(\Omega_{t}^{\Delta}\right)},
\end{aligned}
$$

where matrix $Q=B+B^{T}-\sum_{k=1}^{d} \partial_{k} A_{k}$.

Proof. From (2.13)-(2.14), we have

$$
\begin{array}{r}
\left(\mathcal{L}_{t} \mathbf{w}, \mathbf{w}\right)_{K}=\frac{1}{2}(Q \mathbf{w}, \mathbf{w})_{K}+\frac{1}{2}\left\langle\mathcal{N}_{\mathbf{n}} \mathbf{w}, \mathbf{w}\right\rangle_{\partial K}, \\
\left\langle\mathcal{N}_{\mathbf{n}} \mathbf{w}, \mathbf{w}\right\rangle_{\partial K} \cap \Gamma_{0}+\langle(M-D) \mathbf{w}, \mathbf{w}\rangle_{\partial K} \cap \Gamma_{0}=\langle M \mathbf{w}, \mathbf{w}\rangle_{\partial K} \cap \Gamma_{0},
\end{array}
$$

and

$$
\begin{aligned}
& \left\langle\mathcal{N}_{\mathbf{n}} \mathbf{w}, \mathbf{w}\right\rangle_{\partial K \backslash \Gamma_{0}}+\left\langle\left(A_{0}-\mathcal{N}_{\mathbf{n}}\right)[\mathbf{w}], \mathbf{w}\right\rangle_{\partial K \backslash \Gamma_{0}} \\
= & \left\langle A_{0}[\mathbf{w}], \mathbf{w}\right\rangle_{\partial K \backslash \Gamma_{0}}+\left\langle\mathcal{N}_{\mathbf{n} \mathbf{w}^{-}}, \mathbf{w}\right\rangle_{\partial K \backslash \Gamma_{0}} .
\end{aligned}
$$

Hence, from (3.2), we have the following identity

$$
\begin{aligned}
a_{\Omega_{t}^{\Delta}}(\mathbf{w}, \mathbf{w})= & \frac{1}{2}(Q \mathbf{w}, \mathbf{w})_{\Omega_{t}^{\Delta}}+\frac{1}{2} \sum_{K \in \Omega_{t}^{\Delta}}\langle M \mathbf{w}, \mathbf{w}\rangle_{\Gamma_{0}\left(\Omega_{t}^{\Delta}\right)} \\
& +\frac{1}{2} \sum_{K \in \Omega_{t}^{\Delta}}\left\langle A_{0}[\mathbf{w}], \mathbf{w}\right\rangle_{\partial K \backslash \Gamma_{0}}+\frac{1}{2} \sum_{K \in \Omega_{t}^{\Delta}}\left\langle\mathcal{N}_{\mathbf{n}} \mathbf{w}^{-}, \mathbf{w}\right\rangle_{\partial K \backslash \Gamma_{0}} .
\end{aligned}
$$

Now, let $K$ and $K^{\prime}$ are two adjacent elements with interface $\mathcal{F}_{K}=\partial K \bigcap \partial K^{\prime}$. Since

$$
\begin{aligned}
& \left.\left(\mathbf{w}^{+}-\mathbf{w}^{-}\right) \cdot \mathbf{w}^{+}\right|_{\mathcal{F}_{K} \cap \partial K}+\left.\left(\mathbf{w}^{+}-\mathbf{w}^{-}\right) \cdot \mathbf{w}^{+}\right|_{\mathcal{F}_{K} \cap \partial K^{\prime}} \\
= & \left.\left(\mathbf{w}^{+}-\mathbf{w}^{-}\right) \cdot \mathbf{w}^{+}\right|_{\mathcal{F}_{K} \cap \partial K}+\left.\left(\mathbf{w}^{-}-\mathbf{w}^{+}\right) \cdot \mathbf{w}^{-}\right|_{\mathcal{F}_{K} \cap \partial K} \\
= & {\left.[\mathbf{w}] \cdot[\mathbf{w}]\right|_{\mathcal{F}_{K} \cap \partial K}=\left.[\mathbf{w}] \cdot[\mathbf{w}]\right|_{\mathcal{F}_{K} \cap \partial K^{\prime}}, }
\end{aligned}
$$

then we have

$$
\frac{1}{2} \sum_{K \in \Omega_{t}^{\Delta}}\left\langle A_{0}[\mathbf{w}], \mathbf{w}\right\rangle_{\partial K \backslash \Gamma_{0}}=\frac{1}{4} \sum_{K \in \Omega_{t}^{\Delta}}\left\langle A_{0}[\mathbf{w}],[\mathbf{w}]\right\rangle_{\partial K \backslash \partial \Omega_{t}^{\Delta}}
$$




$$
+\frac{1}{2}\left\langle A_{0}[\mathbf{w}], \mathbf{w}\right\rangle_{\Gamma_{+}\left(\Omega_{t}^{\Delta}\right)}+\frac{1}{2}\left\langle A_{0}[\mathbf{w}], \mathbf{w}\right\rangle_{\Gamma_{-}\left(\Omega_{t}^{\Delta}\right)} .
$$

Next, noting that $\left.\mathbf{n}\right|_{\mathcal{F}_{K} \cap \partial K}=-\left.\mathbf{n}^{\prime}\right|_{\mathcal{F}_{K}} \cap \partial K^{\prime}$ and $\mathcal{N}_{\mathbf{n}}=\mathcal{N}_{\mathbf{n}}^{T}$, we obtain

$$
\begin{aligned}
\frac{1}{2} \sum_{K \in \Omega_{t}^{\Delta}}\left\langle\mathcal{N}_{\mathbf{n}} \mathbf{w}^{-}, \mathbf{w}\right\rangle_{\partial K \backslash \Gamma_{0}} & =\frac{1}{2}\left\langle\mathcal{N}_{\mathbf{n}} \mathbf{w}^{-}, \mathbf{w}\right\rangle_{\Gamma_{ \pm}\left(\Omega_{t}^{\Delta}\right)} \\
& =\frac{1}{2}\left\langle A_{0} \mathbf{w}^{-}, \mathbf{w}\right\rangle_{\Gamma_{+}\left(\Omega_{t}^{\Delta}\right)}-\frac{1}{2}\left\langle A_{0} \mathbf{w}^{-}, \mathbf{w}\right\rangle_{\Gamma_{-}\left(\Omega_{t}^{\Delta}\right)} .
\end{aligned}
$$

Combining (3.4)-(3.6), we arrive at the conclusion of Lemma 3.1.

From Lemma 3.1, we immediately obtain the following result.

Lemma 3.2. Let $\left.\mathbf{u}_{h}^{-}\right|_{\Gamma_{-}\left(\Omega_{t}^{\Delta}\right)}$ be given. Then the solution of discrete problem (3.1) uniquely exists on subdividing domain $\Omega_{t}^{\Delta}$ and satisfies the following stability estimate

$$
\begin{aligned}
& \quad \sigma_{0}\left(\mathbf{u}_{h}, \mathbf{u}_{h}\right)_{\Omega_{t}^{\Delta}}+\left\langle M \mathbf{u}_{h}, \mathbf{u}_{h}\right\rangle_{\Gamma_{0}\left(\Omega_{t}^{\Delta}\right)}+\frac{1}{2} \sum_{K \in \Omega_{t}^{\Delta}}\left\langle A_{0}\left[\mathbf{u}_{h}\right],\left[\mathbf{u}_{h}\right]\right\rangle_{\partial K \backslash \partial \Omega_{t}^{\Delta}} \\
& \quad+\left\langle A_{0} \mathbf{u}_{h}, \mathbf{u}_{h}\right\rangle_{\Gamma_{+}\left(\Omega_{t}^{\Delta}\right)}+\frac{1}{2}\left\langle A_{0} \mathbf{u}_{h}, \mathbf{u}_{h}\right\rangle_{\Gamma_{-}\left(\Omega_{t}^{\Delta}\right)} \\
& \leq \frac{1}{\sigma_{0}}\|\mathbf{f}\|_{\Omega_{t}^{\Delta}}^{2}+4\left\langle A_{0} \mathbf{u}_{0}, \mathbf{u}_{0}\right\rangle_{\Gamma_{-}\left(\Omega_{t}^{\Delta}\right) \cap \Gamma_{-}}+4\left\langle A_{0} \mathbf{u}_{h}^{-}, \mathbf{u}_{h}^{-}\right\rangle_{\Gamma_{-}\left(\Omega_{t}^{\Delta}\right)} .
\end{aligned}
$$

Proof. Since the equation (3.1) can be formulated as a linear algebraic system on subdomain $\Omega_{t}^{\Delta}$, we only need to derive stability estimate (3.7). Taking $\mathbf{v}_{h}=\mathbf{u}_{h}$ in the equation (3.1), and then using Lemma 3.1 and the $\varepsilon$-inequality: $a b \leq \frac{\varepsilon}{2} a^{2}+\frac{1}{2 \varepsilon} b^{2}$ with $\varepsilon=\sigma_{0}, 4$, we imminently obtain estimate (3.7).

Now we are in the position to show the semi-explicit structure of discrete problem (2.17). To this end, we require partition $\mathcal{T}_{h}$ to be made by the following manner. First, we divide the time interval $[0, T]$ into $0=t_{0}<t_{1}<\cdots<$ $t_{N}=T$, next we divide each subdomain $\Omega_{t_{j}}^{\Delta}=\left(t_{j-1}, t_{j}\right] \times \Omega$ into shape-regular meshes, and then the partition $\mathcal{T}_{h}$ is formed by setting (see Fig. 1).

$$
\mathcal{T}_{h}=\bigcup\left\{K: K \in \Omega_{t_{j}}^{\Delta}, j=1, \ldots, N\right\}
$$

Theorem 3.1. Assume that $\mathcal{T}_{h}$ is a shape-regular partition made by (3.8). Then, the discrete problem (2.17) can be solved semi-explicitly, subdomain by subdomain, in the order of $\Omega_{t_{1}}^{\Delta}, \ldots, \Omega_{t_{N}}^{\Delta}$.

Proof. Taking $\Omega_{t}^{\Delta}=\Omega_{t_{j}}^{\Delta}$ in equation (3.1) and noting that $\mathcal{N}_{\mathbf{n}}=A_{0}$ on $\Gamma_{+}\left(\Omega_{t_{j}}^{\Delta}\right)$, we see that the solution $\mathbf{u}_{h}$ among $\left\{\Omega_{t_{j}}^{\Delta}\right\}$ is only coupled via boundaries $\Gamma_{-}\left(\Omega_{t_{j}}^{\Delta}\right)$. Since $\left.\left.\mathbf{u}_{h}^{-}\right|_{\Gamma_{-}\left(\Omega_{t_{1}}^{\Delta}\right)}=\left.\mathbf{u}_{h}^{-}\right|_{\Gamma_{-}}=0,\left.\mathbf{u}_{h}^{-}\right|_{\Gamma_{-}\left(\Omega_{t_{j}}^{\Delta}\right)}=\left.\mathbf{u}_{h}\right|_{\Gamma_{+}\left(\Omega_{t_{j-1}}\right.}\right)$, therefor, by Lemma 3.2, the discrete problem (2.17) or (3.1) can be solved semi-explicitly, in the order of $\Omega_{t_{1}}^{\Delta}, \ldots, \Omega_{t_{N}}^{\Delta}$. 



Figure 1. The two dimensional space-time domain with subdividing domains $\left\{\Omega_{t_{j}}^{\Delta}\right\}$.

\section{Error estimate}

In this section, we will give the error estimates for the DG approximation (2.17).

Let $0<t_{j} \leq T$ and $\Omega_{t_{j}}=\left(0, t_{j}\right] \times \Omega$ be a subdividing domain of $\mathcal{T}_{h}$. In the semi-explicit situation, we may take $\Omega_{t_{j}}=\bigcup_{i=1}^{j} \Omega_{t_{i}}^{\Delta}$ for some $0<j \leq N$. Let $\mathbf{u} \in\left[H^{1}\left(\Omega_{T}\right)\right]^{m}$ be the solution of problem (2.1)-(2.3). From equations (2.16)-(2.17), we have the error equation:

$$
a_{\Omega_{t_{j}}}\left(\mathbf{u}-\mathbf{u}_{h}, \mathbf{v}_{h}\right)=0, \forall \mathbf{v}_{h} \in V_{h} .
$$

Introduce the local $L_{2}$-projection operator $P_{h}: L_{2}\left(\Omega_{T}\right) \rightarrow S_{h}$, restricted to $K \in \mathcal{T}_{h}, P_{h} u \in S_{k}(K)$ satisfies

$$
\left(u-P_{h} u, v\right)_{K}=0, \forall v \in S_{k}(K), K \in \mathcal{T}_{h},
$$

where $S_{k}(K)$ is the local finite element space; see $(2.10)$. Obviously $P_{h}$ is a linear continuous operator from $H^{k+1}(K)$ into $S_{k}(K)$ and $P_{h} v=v$ for all $v \in P_{k}(K) \subset S_{k}(K)$. Hence, by the interpolation theory of Sobolev space [2], we have the standard approximation result

$$
\begin{aligned}
& \left\|u-P_{h} u\right\|_{L_{2}(K)}+h_{K}\left\|u-P_{h} u\right\|_{H^{1}(K)}+h_{K}^{\frac{1}{2}}\left\|u-P_{h} u\right\|_{L_{2}(\partial K)} \\
\leq & C h_{K}^{k+1}|u|_{H^{k+1}(K)}, k \geq 0, \quad K \in \mathcal{T}_{h},
\end{aligned}
$$

where $C$ is a constant independent of element $K$.

First let us consider the partition $\mathcal{T}_{h}$ made of triangle meshes and $S_{k}(K)=$ $P_{k}(K)$. In order to do the error analysis, we still need to introduce a special projection mapping $H^{1}\left(\mathcal{T}_{h}\right)$ into $S_{h}$. For $u \in H^{1}\left(\mathcal{T}_{h}\right)$, the projection function $\mathcal{P} u \in S_{h}$ is defined by finding $\mathcal{P} u \in P_{k}(K)$ such that, for $K \in \mathcal{T}_{h}$,

$$
\begin{gathered}
\int_{K}(u-\mathcal{P} u) v d x=0, \quad \forall v \in P_{k-1}(K), \\
\int_{\mathcal{F}_{K}}(u-\mathcal{P} u) v d s=0, \quad \forall v \in P_{k}\left(\mathcal{F}_{K}\right),
\end{gathered}
$$


where $\mathcal{F}_{K}$ be some one face of element $K$ and the first condition is vacuous if $k=0$. Bear in mind that although element $K$ may have several faces $\left\{\mathcal{F}_{K}\right\}$, we only select one face to define the projection in (4.5).

Lemma 4.1. The projection function $\mathcal{P} u$ is well posed and satisfies the approximation property

$$
\begin{aligned}
& \|u-\mathcal{P} u\|_{L_{2}(K)}+h_{K}\|u-\mathcal{P} u\|_{H^{1}(K)}+h_{K}^{\frac{1}{2}}\|u-\mathcal{P} u\|_{L_{2}(\partial K)} \\
\leq & C h_{K}^{k+1}|u|_{H^{k+1}(K)}, k \geq 0, \quad K \in \mathcal{T}_{h},
\end{aligned}
$$

where $C$ is a constant independent of element $K$.

Proof. Let us begin by proving the unique existence of function $\mathcal{P} u \in P_{k}(K)$ satisfying (4.4)-(4.5). Since

$$
\operatorname{dim}\left(P_{k-1}(K)\right)+\operatorname{dim}\left(P_{k}\left(\mathcal{F}_{K}\right)=C_{d}^{k-1+d}+C_{d-1}^{k+d-1}=C_{d}^{k+d}=\operatorname{dim}\left(P_{k}(K)\right),\right.
$$

we see that the linear system (4.4)-(4.5) is square so that we only need to show that $\mathcal{P} u=0$ if $u=0$. Without loss of generality, we assume that the face $\mathcal{F}_{K}$ in (4.5) lies on the hyperplane $x_{1}=0$ and $x_{1}<0$ when $x \in K$ (otherwise we may use the affine transformation $F: K \rightarrow \hat{K}$ such that $\mathcal{F}_{\hat{K}}$ lies on $\hat{x}_{1}=0$, and $\hat{x}_{1}<0$ when $\left.\hat{x} \in \hat{K}\right)$. Let $u=0$, then we have from (4.5) that $\left.\mathcal{P} u\right|_{\mathcal{F}_{K}}=0$ and hence $\mathcal{P} u=x_{1} p$ for some polynomial $p \in P_{k-1}(K)$. Taking $v=p$ in (4.4), we get

$$
\left(x_{1} p, p\right)_{K}=\left(x_{1}, p^{2}\right)_{K}=0,
$$

since $x_{1}<0$ on $K$, we conclude that $p=0$. This implies that $\mathcal{P} u=0$ on $K$.

Now we are in the position to prove the approximation property (4.6). Let $P_{h}$ be the $L_{2}$-projection defined by (4.2). From (4.5) we see that

$$
\left\langle\mathcal{P} u-P_{h} u, \mathcal{P} u-P_{h} u\right\rangle_{\mathcal{F}_{K}}=\left\langle u-P_{h} u, \mathcal{P} u-P_{h} u\right\rangle_{\mathcal{F}_{K}},
$$

hence

$$
\left\|\mathcal{P} u-P_{h} u\right\|_{L_{2}\left(\mathcal{F}_{K}\right)} \leq\left\|u-P_{h} u\right\|_{L_{2}\left(\mathcal{F}_{K}\right)} .
$$

Introduce the polynomial space

$$
P_{k}^{0}(K)=\left\{v \in P_{k}(K):(v, p)_{K}=0, \forall p \in P_{k-1}(K)\right\} .
$$

It is easy to see that $\|\cdot\|_{L_{2}\left(\mathcal{F}_{K}\right)}$ defines a norm on space $P_{k}^{0}(K)$ (see the argument of the unique existence) and this norm is equivalent to norm $\|\cdot\|_{L_{2}(K)}$, since $P_{k}^{0}(K)$ is a finite dimensional space. Then, by a simple scaling argument, we have

$$
\|v\|_{L_{2}(K)} \leq C h_{K}^{\frac{1}{2}}\|v\|_{L_{2}\left(\mathcal{F}_{K}\right)}, \forall v \in P_{k}^{0}(K),
$$

hence, noting that $\mathcal{P} u-P_{h} u \in P_{k}^{0}(K)$, it implies from (4.7) that

$$
\left\|\mathcal{P} u-P_{h} u\right\|_{L_{2}(K)} \leq C h_{K}^{\frac{1}{2}}\left\|\mathcal{P} u-P_{h} u\right\|_{L_{2}\left(\mathcal{F}_{K}\right)} \leq C h_{K}^{\frac{1}{2}}\left\|u-P_{h} u\right\|_{L_{2}\left(\mathcal{F}_{K}\right)} .
$$


Hence, by using the triangle inequality and approximation property (4.3), we obtain the estimate of $\|u-\mathcal{P} u\|_{L_{2}(K)}$. Furthermore, by using the finite element inverse inequality

$$
h_{K}\|v\|_{H^{1}(K)}+h_{K}^{\frac{1}{2}}\|v\|_{L_{2}(\partial K)} \leq C\|v\|_{L_{2}(K)}, \forall v \in P_{k}(K), \quad K \in \mathcal{T}_{h} .
$$

(taking $v=\mathcal{P} u-P_{h} u$ ) and approximation property (4.3), we complete the proof.

In what follows, for vector function $\mathbf{u}$, we set $P_{h} \mathbf{u}=\left(P_{h} u_{1}, \ldots, P_{h} u_{m}\right)^{T}$, $\mathcal{P} \mathbf{u}=\left(\mathcal{P} u_{1}, \ldots, \mathcal{P} u_{m}\right)^{T}$. We also denote by $w^{c}$ the piecewise constant approximation of function $w$ defined by

which has the approximation property

$$
\left.w^{c}\right|_{K}=\frac{1}{|K|} \int_{K} w, \forall K \in \mathcal{T}_{h}
$$

$$
\left\|w-w^{c}\right\|_{0, \infty, K} \leq C h_{K}\|w\|_{1, \infty, K}, \quad \forall K \in \mathcal{T}_{h} .
$$

For the error analysis, we still need an additional assumption on the partition $\mathcal{T}_{h}$,

$$
\text { Each element } K \in \mathcal{T}_{h} \text { at most has one face } \mathcal{F}_{K} \text { lying on } \Gamma_{0} \text { and }
$$$$
\text { this face (if exist) is used in the interpolation condition (4.5). }
$$

Introduce the DG-norm

$$
\begin{aligned}
\|\| \mathbf{u}\|\|_{\Omega_{t_{j}}}^{2}= & \sigma_{0}\|\mathbf{u}\|_{\Omega_{t_{j}}}^{2}+\sum_{K \in \Omega_{t_{j}}}\left\langle A_{0}[\mathbf{u}],[\mathbf{u}]\right\rangle_{\partial K \backslash \partial \Omega_{t_{j}}} \\
& +\left\langle A_{0} \mathbf{u}, \mathbf{u}\right\rangle_{\Gamma_{+}\left(\Omega_{t_{j}}\right)}+\left\langle A_{0} \mathbf{u}, \mathbf{u}\right\rangle_{\Gamma_{-}\left(\Omega_{t_{j}}\right)}+\langle M \mathbf{u}, \mathbf{u}\rangle_{\Gamma_{0}\left(\Omega_{t_{j}}\right)} .
\end{aligned}
$$

Now we can state our error estimate result.

Theorem 4.1. Assume that $\mathcal{T}_{h}$ is a shape-regular partition made of triangle meshes and $S_{k}(K)=P_{k}(K)$. Further, assume that condition (4.10) holds, and let $\mathbf{u}$ and $\mathbf{u}_{h}$ be the solutions of problems (2.1)-(2.3) and (2.17), respectively. Then we have

$$
\|\left|\mathbf{u}-\mathbf{u}_{h}\right|||_{\Omega_{t_{j}}} \leq C h^{k+\frac{1}{2}}|\mathbf{u}|_{H^{k+1}\left(\Omega_{t_{j}}\right)}, k \geq 0 .
$$

Proof. First we decompose the error by setting

$$
\mathbf{u}-\mathbf{u}_{h}=\mathbf{u}-\mathcal{P} \mathbf{u}+\mathcal{P} \mathbf{u}-\mathbf{u}_{h}=\boldsymbol{\eta}+\boldsymbol{\theta} .
$$

From Lemma 3.1 and error equation (4.1), we obtain

$$
\begin{aligned}
\frac{1}{4}\left\|\left|\mathbf{u}-\mathbf{u}_{h} \|\right|_{\Omega_{t_{j}}}^{2} \leq\right. & a_{\Omega_{t_{j}}}\left(\mathbf{u}-\mathbf{u}_{h}, \mathbf{u}-\mathbf{u}_{h}\right)=a_{\Omega_{t_{j}}}\left(\mathbf{u}-\mathbf{u}_{h}, \mathbf{u}-\mathcal{P} \mathbf{u}\right) \\
= & \sum_{K \in \Omega_{t_{j}}}\left(\mathcal{L}_{t}\left(\mathbf{u}-\mathbf{u}_{h}\right), \boldsymbol{\eta}\right)_{K} \\
& +\frac{1}{2} \sum_{K \in \Omega_{t_{j}}}\left\langle\left(A_{0}-\mathcal{N}_{\mathbf{n}}\right)\left[\mathbf{u}-\mathbf{u}_{h}\right], \boldsymbol{\eta}\right\rangle_{\partial K \backslash \Gamma_{0}}
\end{aligned}
$$




$$
\begin{aligned}
& +\frac{1}{2}\left\langle(M-D)\left(\mathbf{u}-\mathbf{u}_{h}\right), \boldsymbol{\eta}\right\rangle_{\Gamma_{0}\left(\Omega_{t_{j}}\right)} \\
= & T_{1}+T_{2}+T_{3} .
\end{aligned}
$$

Now let us estimate terms $T_{i}, i=1,2,3$. Since

$$
\begin{aligned}
T_{1} & =\sum_{K \in \Omega_{t_{j}}}\left(\mathcal{L}_{t} \boldsymbol{\theta}, \boldsymbol{\eta}\right)_{K}+\sum_{K \in \Omega_{t_{j}}}\left(\mathcal{L}_{t} \boldsymbol{\eta}, \boldsymbol{\eta}\right)_{K} \\
& =\sum_{K \in \Omega_{t_{j}}}\left(\left(A_{0} \partial_{t}+\sum_{k=0}^{d} A_{k} \partial_{k}\right) \boldsymbol{\theta}, \boldsymbol{\eta}\right)_{K}+\sum_{K \in \Omega_{t_{j}}}(B \boldsymbol{\theta}, \boldsymbol{\eta})_{K}+\sum_{K \in \Omega_{t_{j}}}\left(\mathcal{L}_{t} \boldsymbol{\eta}, \boldsymbol{\eta}\right)_{K},
\end{aligned}
$$

then by the definition of $\mathcal{P}$ and noting that $A_{0}^{c} \partial_{t} \boldsymbol{\theta}$ and $A_{k}^{c} \partial_{k} \boldsymbol{\theta}$ are in $P_{k-1}(K)$, we obtain

$$
\begin{aligned}
T_{1}= & \sum_{K \in \Omega_{t_{j}}}\left(\left(\left(A_{0}-A_{0}^{c}\right) \partial_{t}+\sum_{k=0}^{d}\left(A_{k}-A_{k}^{c}\right) \partial_{k}\right) \boldsymbol{\theta}, \boldsymbol{\eta}\right)_{K} \\
& +\sum_{K \in \Omega_{t_{j}}}(B \boldsymbol{\theta}, \boldsymbol{\eta})_{K}+\sum_{K \in \Omega_{t_{j}}}\left(\mathcal{L}_{t} \boldsymbol{\eta}, \boldsymbol{\eta}\right)_{K} \\
\leq & C\left\|h_{K} \boldsymbol{\theta}\right\|_{1, \Omega_{t_{j}}}\|\boldsymbol{\eta}\|_{\Omega_{t_{j}}}+|B|_{\infty}\|\boldsymbol{\theta}\|_{\Omega_{t_{j}}}\|\boldsymbol{\eta}\|_{\Omega_{t_{j}}}+C\|\boldsymbol{\eta}\|_{1, \Omega_{t_{j}}}\|\boldsymbol{\eta}\|_{\Omega_{t_{j}}} \\
\leq & C\|\boldsymbol{\theta}\|_{\Omega_{t_{j}}}\|\boldsymbol{\eta}\|_{\Omega_{t_{j}}}+C\|\boldsymbol{\eta}\|_{1, \Omega_{t_{j}}}\|\boldsymbol{\eta}\|_{\Omega_{t_{j}}} \\
\leq & C\left(\|\boldsymbol{\eta}\|_{\Omega_{t_{j}}}^{2}+\|\boldsymbol{\eta}\|_{1, \Omega_{t_{j}}}\|\boldsymbol{\eta}\|_{\Omega_{t_{j}}}\right)+\frac{1}{16}\left\|\mathbf{u}-\mathbf{u}_{h} \mid\right\|_{\Omega_{t_{j}}}^{2},
\end{aligned}
$$

where we have used the approximation property (4.9) and the inverse inequality (4.8).

Next, we write

$$
T_{3}=\frac{1}{2}\langle(M-D) \boldsymbol{\theta}, \boldsymbol{\eta}\rangle_{\Gamma_{0}\left(\Omega_{t_{j}}\right)}+\frac{1}{2}\langle(M-D) \boldsymbol{\eta}, \boldsymbol{\eta}\rangle_{\Gamma_{0}\left(\Omega_{t_{j}}\right)} .
$$

Since $\left(M^{c}-D^{c}\right) \boldsymbol{\theta} \in P_{k}\left(\mathcal{F}_{K}\right)$ on element face $\mathcal{F}_{K} \in \Gamma_{0}\left(\Omega_{t_{j}}\right) \subset \Gamma_{0}$, then by using (4.5) and assumption (4.10) to obtain

$$
\begin{aligned}
T_{3} & =\frac{1}{2}\left\langle\left(M-D-\left(M^{c}-D^{c}\right)\right) \boldsymbol{\theta}, \boldsymbol{\eta}\right\rangle_{\Gamma_{0}\left(\Omega_{t_{j}}\right)}+\frac{1}{2}\langle(M-D) \boldsymbol{\eta}, \boldsymbol{\eta}\rangle_{\Gamma_{0}\left(\Omega_{t_{j}}\right)} \\
& \leq \frac{1}{2}|M-D|_{1, \infty}\left\|h_{K} \boldsymbol{\theta}\right\|_{L_{2}\left(\Gamma_{0}\left(\Omega_{t_{j}}\right)\right)}\|\boldsymbol{\eta}\|_{L_{2}\left(\Gamma_{0}\right)}+\frac{1}{2}|M-D|_{\infty}\|\boldsymbol{\eta}\|_{L_{2}\left(\Gamma_{0}\right)}^{2} \\
& \leq C\|\boldsymbol{\theta}\|_{\Omega_{t_{j}}}\|\boldsymbol{\eta}\|_{L_{2}\left(\Gamma_{0}\right)}+\frac{1}{2}|M-D|_{\infty}\|\boldsymbol{\eta}\|_{L_{2}\left(\Gamma_{0}\right)}^{2}
\end{aligned}
$$

Finally, we need to estimate

$$
T_{2}=\frac{1}{2} \sum_{K \in \Omega_{t_{j}}}\left\langle\left(A_{0}-\mathcal{N}_{\mathbf{n}}\right)\left[\mathbf{u}-\mathbf{u}_{h}\right], \boldsymbol{\eta}\right\rangle_{\partial K \backslash \partial \Omega_{t_{j}}}+\left\langle A_{0}\left(\mathbf{u}-\mathbf{u}_{h}\right), \boldsymbol{\eta}\right\rangle_{\Gamma_{-}\left(\Omega_{t_{j}}\right)} .
$$


By using the Cauchy inequality and noting that $A_{0}>0$, we immediately obtain

$$
T_{2} \leq C \sum_{K \in \Omega_{t_{j}}}\|\boldsymbol{\eta}\|_{L_{2}(\partial K)}^{2}+\frac{1}{16}\|\| \mathbf{u}-\mathbf{u}_{h} \|\left.\right|_{\Omega_{t_{j}}} ^{2} .
$$

Now, substituting the estimates of $T_{1}, T_{2}$ and $T_{3}$ into (4.12), and using approximation property (4.6) to estimate $\boldsymbol{\eta}=\mathbf{u}-\mathcal{P} \mathbf{u}$, we arrive at the conclusion of Theorem 4.1.

Let us emphasize that the error estimate in Theorem 4.1 is optimal for DG methods within quasi-regular meshes [11]. However, for a scalar hyperbolic equation, this order of convergence can be further improved if some special structured meshes are used, see $[4,12]$.

In the above estimate of $T_{3}$, the projection $\mathcal{P}$ and assumption (4.10) play a major role. But, it is easy to see that for rectangular meshes and $d \geq 2$, there must be a boundary element which at least has two faces lying on $\Gamma_{0}$ such that condition (4.10) is violated. In order to make the error estimate to be applicable to the partitions including rectangular meshes, we need to present a new assumption instead of (4.10). We assume that the boundary matrix $M$ satisfies, for any element face $\mathcal{F}_{K} \in \Gamma_{0}$,

$$
\left|\langle(M-D) \mathbf{w}, \mathbf{v}\rangle_{\mathcal{F}_{K}}\right| \leq C_{M}\langle M \mathbf{w}, \mathbf{w}\rangle_{\mathcal{F}_{K}}^{\frac{1}{2}}\langle\mathbf{v}, \mathbf{v}\rangle_{\mathcal{F}_{K}}^{\frac{1}{2}}, \quad \forall \mathbf{w}, \mathbf{v} \in\left[L_{2}\left(\Gamma_{0}\right)\right]^{m}
$$

where $C_{M}$ is a constant independent of $\mathbf{w}$ and $\mathbf{v}$. In fact, for many physics problems, we may choose the boundary matrix $M$ properly such that both the boundary value condition of the problem and assumption (4.16) can be satisfied meanwhile. For example, the boundary matrix $M$ selected carefully by us in the example of wave equation (see Section 2) satisfies the assumption (4.16) with $C_{M}=\sqrt{5}$.

Theorem 4.2. Assume that $\mathcal{T}_{h}$ is a shape-regular partition and condition (4.16) holds, and let $\mathbf{u}$ and $\mathbf{u}_{h}$ be the solutions of problems (2.1)-(2.3) and (2.17), respectively. Then we have

$$
\|\left.\left|\mathbf{u}-\mathbf{u}_{h}\right|\right|_{\Omega_{t_{j}}} \leq C h^{k+\frac{1}{2}}|\mathbf{u}|_{H^{k+1}\left(\Omega_{t_{j}}\right)}, k \geq 0 .
$$

Proof. Let $P_{h}$ be the local $L_{2}$-projection operator defined by (4.2). By using $P_{h} \mathbf{u}$ instead of $\mathcal{P} \mathbf{u}$ in the argument of Theorem 4.1, we only need to estimate $T_{3}$ in (4.12). By assumption (4.16) we have

$$
\begin{aligned}
T_{3} & =\frac{1}{2}\left\langle(M-D)\left(\mathbf{u}-\mathbf{u}_{h}\right), \boldsymbol{\eta}\right\rangle_{\Gamma_{0}\left(\Omega_{t_{j}}\right)} \\
& \leq \frac{1}{2} C_{M}\left\langle M\left(\mathbf{u}-\mathbf{u}_{h}\right), \mathbf{u}-\mathbf{u}_{h}\right\rangle_{\Gamma_{0}\left(\Omega_{t_{j}}\right)}^{\frac{1}{2}}\|\boldsymbol{\eta}\|_{L_{2}\left(\Gamma_{0}\right)} \\
& \leq C\|\boldsymbol{\eta}\|_{L_{2}\left(\Gamma_{0}\right)}^{2}+\left.\frac{1}{16}\left\|\mathbf{u}-\mathbf{u}_{h}\right\|\right|_{\Omega_{t_{j}}} ^{2} .
\end{aligned}
$$

This is the same estimate as (4.14). The remainder argument is completely similar to that of Theorem 4.1 . 
In Theorem 4.1 and Theorem 4.2, if we take $\Omega_{t_{j}}=\Omega_{T}$, we immediately obtain the global error estimates.

\section{Numerical experiments}

In this section, we will present some numerical results to show the validity of our method. Let us consider the wave equation in two-dimensional domain, written as a first order hyperbolic system as that in Section 2 . We take $\Omega=$ $[0,2 \pi]^{2}$ and the exact solution

$$
u_{0}=e^{t} \sin x \sin y, u_{1}=e^{t} \cos x \sin y, u_{2}=e^{t} \sin x \cos y .
$$

In our numerical experiments, we partition the space-time domain $\Omega_{T}$ into regular rectangular meshes of size $h=1 / 2^{l}$ and use the $Q_{1}$-finite element. The numerical results are given in Table 5.1, in which the $L_{2}$ - errors are presented at $t=1$ and $t=2$, respectively, for successively halving mesh size $h$. The numerical convergence rate is computed by using the formula $\alpha=\ln \left(e_{h} / e_{\frac{h}{2}}\right) / \ln 2$, where $e_{h}$ represents the error between the exact solution and the DG solution in the $L_{2}$-norm with mesh size $h$. We see that an $\mathcal{O}\left(h^{2}\right)$ rate of convergence is observed, in contrast to our theoretical estimate of $\mathcal{O}\left(h^{1.5}\right)$. The $h^{1 / 2}$ gap between theoretical and actual convergence rates is typical for the DG methods.

Table 5.1 Error and convergence rate

\begin{tabular}{|c|lr|lr|}
\hline & \multicolumn{2}{|c|}{$\left\|\mathbf{u}-\mathbf{u}_{h}\right\|_{t=1}$} & \multicolumn{2}{c|}{$\left\|\mathbf{u}-\mathbf{u}_{h}\right\|_{t=2}$} \\
mesh $h$ & error & rate & error & rate \\
\hline $1 / 8$ & 0.3442 & - & 0.4532 & - \\
$1 / 16$ & $0.878 \mathrm{e}-1$ & 1.971 & $1.178 \mathrm{e}-1$ & 1.944 \\
$1 / 32$ & $0.198 \mathrm{e}-1$ & 2.146 & $0.285 \mathrm{e}-1$ & 2.048 \\
$1 / 64$ & $0.489 \mathrm{e}-2$ & 2.019 & $0.698 \mathrm{e}-2$ & 2.028 \\
$1 / 128$ & $0.121 \mathrm{e}-2$ & 2.005 & $0.174 \mathrm{e}-2$ & 2.002 \\
\hline
\end{tabular}

Acknowledgements. The authors would like to thank the anonymous referees for many helpful suggestions which improved the presentation of this paper.

\section{References}

[1] D. N. Arnold, F. Brezzi, and B. Cockburn, et al., Unified analysis of discontinuous Galerkin methods for elliptic problems, SIAM J. Numer. Anal. 39 (2001), no. 5, 17491779.

[2] P. G. Cairlet, The Finite Element Methods for Elliptic Problems, North-Holland Publish, Amsterdam, 1978.

[3] B. Cockburn, G. E. Karniadakis, and C. W. Shu, Discontinuous Galerkin Methods, Theory, Computation and Applications, Lecture Notes Comput. Sci. Eng., Vol. 11, SpringerVerlag, Berlin, 2000.

[4] B. Cockburn, B. Dong, and J. Guzmán, Optimal convergence of the original DG method for the transport-reaction equation on special meshes, SIAM J. Numer. Anal. 46 (2008), no. $3,1250-1265$. 
[5] B. Cockburn and C. W. Shu, Runge-Kutta discontinuous Galerkin methods for convection-dominated problems (review article), J. Sci. Compu. 16 (2001), no. 3, 173261.

[6] A. Ern and J. L. Guermond, Discontinuous Galerkin methods for Friedrichs' systems, I. general theory, SIAM J. Numer. Anal. 44 (2006), no. 2, 753-778.

[7] R. S. Falk and G. R. Richter, Explicit finite element methods for symmetric hyperbolic equations, SIAM J. Numer. Anal. 36 (1999), no. 3, 935-952.

[8] K. Friedrichs, Symmetric positive linear differential equations, Comm. Pure Appl. Math. 11 (1958), 333-418.

[9] C. Johnson, U. Nävert, and J. Pitkäranta, Finite element methods for linear hyperbolic problems, Comput. Methods Appl. Mech. Engrg. 45 (1984), no. 1-3, 285-312.

[10] P. Monk and G. R. Richter, A discontinuous Galerkin method for linear symmetric hyperbolic systems in inhomogeneous media, J. Sci. Compu. 22 (2005), 443-477.

[11] T. E. Peterson, A note on the convergence of the discontinuous Galerkin method for a scalar hyperbolic equation, SIAM J. Numer. Anal. 28 (1991), no. 1, 133-140.

[12] G. Richter, An optimal-order error estimate for discontinuous Galerkin method, Math. Comp. 50 (1988), 75-88.

[13] R. Winther, Astable finite element method for first-order hyperbolic systems, Math. Comp. 36 (1981), 65-86.

[14] T. Zhang, Discontinuous Finite Element Theory and Method, Sincece Press, Beijing, 2012.

TiE ZHANG

Department of Mathematics and the State Key Laboratory of Synthetical Automation for Process Industries

NORTHEASTERN UNIVERSITY

Shenyang 110004, P. R. China

E-mail address: ztmath@163.com

JiNGNA LiU

Department of Mathematics and the State Key Laboratory of

Synthetical Automation for Process Industries

NORTHEASTERN UNIVERSITY

Shenyang 110004, P. R. China

E-mail address: jingna0810@163.com 